\title{
LITERATURBERICHT
}

\section{Informell oder institutionalisiert? Die Internationalisierung der inneren Sicherheit}

\author{
Eva Herschinger / Markus Jachtenfuchs
}

\begin{abstract}
Informal or Institutionalized? The Internationalization of Internal Security
Abstract: Although international police cooperation is not a new phenomenon and despite its relevance for the monopoly of force, studies applying a political science or international relations (IR) perspective are scarce. Through a 'competition of claims', this article reviews and organizes current literature on cooperation in internal security to highlight crucial points of entry for IR- and political science approaches. While both claims argue along the lines of functionalist regime theory, the 'informality claim' stresses state's interest to uphold autonomy and their accordant preference for informal cooperation. In contrast, the 'institutionalization claim' underscores the willingness of states to create formal and strong institutions as they promise efficiency gains in light of transborder problems. By focusing on central issues in police cooperation (terrorism, drugs, money laundering, organized crime) this review highlights that internal security can become a prospering field for IR- and political science theorizing.
\end{abstract}

Schlagwörter: innere Sicherheit, internationale Kooperation, internationale Institutionen, Informalität, Geschichte der Polizeikooperation

Keywords: Internal security, International cooperation, International institutions, Informality, History of police cooperation

\section{Einleitung}

Einen Literaturbericht über „die Internationalisierung der inneren Sicherheit“ schreiben zu wollen, wirkt zunächst wie ein paradoxes Unterfangen. ${ }^{1}$ Innere Sicherheit erscheint klar von der internationalen Sphäre getrennt, und diese Trennung lässt sich systematisch wie historisch begründen. Systematisch geht es bei der inneren Sicherheit um Herrschaftssicherung im Innern und um die Nutzung des staatlichen Gewaltmonopols (ausgeübt durch die Polizei), das für Max Weber und andere nicht nur eine entscheidende Ressource moderner Staatlichkeit darstellt, sondern auch das zentrale Merkmal, durch das sich der Staat von allen anderen Formen politischer Organisation unterscheidet (Weber 1980, S. 29). Es ist deshalb plausibel anzunehmen, dass es im institutionellen Eigeninteresse von

1 Wir danken der PVS-Redaktion für ihre konstruktiven Kommentare. 
Staaten ist, diesen Bereich ohne Beeinflussung durch andere Staaten, Einschränkungen durch internationale Verträge oder Aktivitäten internationaler Organisationen organisieren zu können.

Auch historisch spricht vieles für diese Trennung. Studien über die Entstehung nationaler Polizeien zeigen eine extreme Pfadabhängigkeit der Binnenentwicklung dieser Systeme und die weitgehende Abwesenheit externer Einflüsse (vgl. z. B. Boldt 2007; Emsley 1996; Knöbl 2008). Selbst den alliierten Besatzungsmächten in Deutschland gelang es nicht, nachhaltig Elemente ihrer eigenen Polizeisysteme in Deutschland zu verankern (Lange 1999, Kap. 3). Vergleichende Analysen von Polizeisystemen identifizieren nicht allzu viele Ähnlichkeiten, sondern zeichnen ein außerordentlich buntes Bild von Institutionen und Organisationen. Die „varieties of policing“ scheinen die „varieties of capitalism“ an Vielfalt deutlich zu übertreffen (Bayley 1985).

Trotzdem kooperieren Staaten schon seit Ende des 19. Jahrhundert im Bereich der inneren Sicherheit. Mittlerweile hat diese Kooperation an Intensität und inhaltlicher Breite stark zugenommen. Hierzu existiert eine lange Zeit auf wenige Autoren beschränkte Literatur, die aber in den letzten Jahren stark angewachsen ist. Diese Literatur ist allerdings zumeist höchst spezialisiert, an den Prioritäten der politischen Entscheidungsträger ausgerichtet und häufig von Polizeipraktikern verfasst. Vielfach ist sie deskriptiv und hängt in ihren Analysen den dramatischen politischen Entwicklungen in der EU oder der UN seit 9/11 hinterher. Die wissenschaftlich orientierte Literatur wird häufig von Juristen, Kriminologen oder Soziologen verfasst und ist deshalb nicht an politikwissenschaftlichen Fragestellungen orientiert.

All dies muss kein Nachteil sein. Es bleibt aber der Befund, dass zwar ein beträchtliches Ausmaß an internationaler Kooperation im Bereich der inneren Sicherheit existiert, diese aber kaum aus der Perspektive der Politikwissenschaft oder der Internationalen Beziehungen (IB) analysiert wird, obwohl diese Kooperation politisch wie wissenschaftlich wichtig ist, weil sie den Kernbereich von Staatlichkeit betrifft.

Im folgenden Literaturbericht wählen wir deshalb eine etwas ungewöhnliche Vorgehensweise. Wir schildern keine tatsächlichen theoretischen, methodischen oder empirischen Kontroversen zwischen den Autorinnen und Autoren. Stattdessen reinterpretieren wir die existierende Literatur aus dem Blickwinkel zentraler IB-Theorien, um die Literatur überhaupt für die Internationalen Beziehungen im Speziellen und für die Politikwissenschaft im Allgemeinen zugänglich zu machen und um ein IB-Mainstreaming dieser Literatur zu fördern. Dazu konstruieren wir auf der Basis der vorliegenden Arbeiten zwei stilisierte Thesen, die Informalitätsthese und die Institutionalisierungsthese und ordnen ihr die Literatur jeweils zu.

Beide Thesen argumentieren ähnlich der funktionalistischen Regimetheorie (Keohane 1984), auf die sich die Literatur aber kaum je bezieht: Internationale Zusammenarbeit hegt grenzüberschreitende Externalitäten ein, erzeugt Erwartungssicherheit und stellt Informationen über das Verhalten anderer bereit. Machtasymmetrien zwischen den Staaten haben einen Einfluss auf die Struktur der Zusammenarbeit. Der Unterschied liegt in der Akzentsetzung: Die Informalitätsthese betont, dass Staaten zwar Effizienzgewinne durch Kooperation realisie- 
ren wollen, ihr Hauptinteresse aber die Aufrechterhaltung staatlicher Handlungsfähigkeit sowohl gegenüber gesellschaftlichen Akteuren, als auch gegenüber internationalen Institutionen ist (ähnlich in den IB Wolf 2000). Aus diesem Grund wird gerade im herrschaftsnahen Bereich der inneren Sicherheit häufig und bewusst informelle Zusammenarbeit favorisiert. Zentrale Akteure sind hier Verwaltungs- und Polizeipraktiker. Die Institutionalisierungsthese hingegen betont vor allem die Effizienzgewinne durch die Schaffung formaler und starker Institutionen, für die Staaten auch bereit sind, Einschränkungen ihrer Autonomie in Kauf zu nehmen. Der Akzent liegt hier eher auf der Wahl zwischen universellen Institutionen mit heterogener Mitgliedschaft und regionalen Institutionen mit homogener Mitgliedschaft. Zentrale Akteure sind politische Entscheidungsträger.

Es geht uns dabei um einen stilisierten Thesenwettbewerb, nicht um einen realen Theorietest. Zudem ergänzen sich die Thesen bei einigen Autoren eher als dass sie sich explizit widersprechen, und die empirische Literatur zeigt, dass Informalisierung und Institutionalisierung lange mit unterschiedlicher Intensität parallel verliefen, sprich, die informelle Zusammenarbeit eine wichtige Voraussetzung für die Verstärkung der formellen Institutionalisierung war und umgekehrt. Diese Parallelität wird jedoch nicht ausreichend in der Literatur reflektiert, die häufig dazu neigt, sich fast ausschließlich entweder auf informelle Kooperation (auf die gerade ältere Studien fokussieren) oder institutionalisierte Zusammenarbeit (dies gilt vor allem für Arbeiten zur EU) zu konzentrieren (Ausnahmen sind Anderson et al. 1995; Den Boer 2005; Walker 2004).

Inhaltlich beschränken wir uns in diesem Bericht auf Literatur, die diejenigen Felder abdeckt, die seit längerer Zeit zum Kernbereich des Politikfeldes innere Sicherheit auf der internationalen Ebene gehören: Terrorismus, Drogenhandel und Geldwäsche. Selbst hier geht es uns nicht um eine enzyklopädische Bearbeitung des Feldes, sondern um die theorie- und thesenorientierte Rekonstruktion großer Trends bei der Internationalisierung der inneren Sicherheit. In der Folge betrachten wir zunächst die Informalitätsthese (Abschnitt 2), danach die Institutionalisierungsthese (Abschnitt 3) und zeigen schließlich die Bezugspunkte beider Thesen sowie die Anschlussfähigkeit der Forschung vor allem für konstruktivistische Ansätze auf (Abschnitt 4).

\section{Die Informalitätsthese: Transgouvernementale Polizistennetzwerke als treibende Kräfte?}

Autoren, die der Informalitätsthese zugeordnet werden können, sind vor allem Didier Bigo, Heiner Busch, James Sheptycki, Ethan Nadelmann und Mathieu Deflem, da sie die Bedeutung von Polizisten oder Verwaltungsmitarbeitern unterhalb der Regierungsebene in bilateralen, informellen und vor allem geheimen Netzwerken betonen. Diese Struktur der Kooperation wird historisch erklärt: Ihre Fundamente wurden im letzten Drittel des 19. Jahrhunderts gelegt (vgl. Busch 1995, S. 256-264; Sheptycki 2002; Gerspacher 2008; Andreas und Nadelmann 2006, S. 62). Vorschläge aus dieser Zeit, Polizeien über Ländergrenzen hinweg zu organisieren, kamen laut dieser Literatur nicht von Politikern, sondern vor allem von 
Praktikern, denen die zunehmende Bedeutung grenzüberschreitender Kriminalität bewusst wurde. Entscheidend waren damals nicht nur persönliche Kontakte, sondern auch die zahlreichen nationalen wie internationalen Polizistenkongresse, auf denen sich um die Jahrhundertwende die Praktiker trafen und mit einer steigenden Zahl an Kriminologen diskutierten - weitgehend ohne Beteiligung politischen Personals (Busch 1995, S. 267). Polizisten nutzen diese Kongresse für den Austausch über politische, bürokratische und rechtliche Hindernisse einer grenzüberschreitenden Zusammenarbeit und über passende transnationale Lösungsmöglichkeiten (Andreas u. Nadelmann 2006, S. 82).

Vor allem zahlreiche Arbeiten von Ethan Nadelmann erweitern dieses Argument auf die Zeit nach dem 2. Weltkrieg. Nadelmann argumentiert, dass Polizeikooperation in dieser Zeit vor allem von den USA vorangetrieben wurde, die informelle Kontakte auf allen Ebenen - vor allem aber bei der Drogenbekämpfung - anstrebten (Nadelmann 1993b; 1990; Andreas u. Nadelmann 2006). Auf diese Weise versuchten die USA, den Geltungsbereich ihrer Rechtsordnung vor allem nach Europa, letztlich aber weltweit auszudehnen und auch direkt operativ in anderen Staaten tätig zu werden (Nadelmann 1995; siehe auch Friman 1996). Zwar machten sich die USA für ein globales Drogenprohibitionsregime innerhalb der UN stark, agierten aber in anderen Bereichen bilateral, informell und ohne Kenntnis der Öffentlichkeit (McAllister 2000; Bewley-Taylor 1999), so bspw. in der Diffusion von investigativen Ermittlungsmethoden wie die Führung von Informanten, verdeckten Ermittlern oder die Sammlung von Intelligence-Daten. Diese einflussreiche Stellung konnten die USA durch ihre weltweite Präsenz von FBI- oder DEA-Beamten (Drug Enforcement Agency) halten (Nadelmann 1993a, bes. Kap. 4).

Während sich Nadelmann vor allem auf die Drogenpolitik der USA konzentriert, arbeitet Didier Bigo in seiner Monographie Police en réseaux (1996) eindrücklich heraus, dass sich die europäische Polizeikooperation nicht durch die Drogenpolitik, sondern durch das Aufkommen des revolutionären Linksterrorismus in Westeuropa Ende der 1960er-Jahre dramatisch intensivierte. Nach seiner These reagierten vor allem hochrangige Polizisten darauf, indem sie sich in etlichen informellen Polizistengremien (u. a. Berner Club, Wiener Club, Police Working Group on Terrorism oder Pompidou Group) zusammenschlossen. Diese Clubs entstanden häufig auf spontane Initiativen von Polizisten hin und dienten als geschütztes Forum für den Austausch über Ermittlungsmethoden, verdächtige Personen und Fälle grenzüberschreitender Kriminalität, die nicht auf politischer Ebene behandelt werden konnten, aber auch der Diskussion von technologischen Innovationen (Bigo 1996, S. 83-84). Unter diesen Zusammenschlüssen war TREVI (entstanden 1975) besonders bedeutsam für die Entwicklung im europäischen Rahmen, da TREVI später im Dritten Pfeiler des Maastrichter Vertrags aufging. Vor allem die Studien von John Benyon und Kollegen versuchen, die zahlreichen Foren zu systematisieren sowie die Entwicklung ihrer Struktur und ihrer Aufgaben über die Zeit zu beschreiben (Benyon, et al. 1993; Benyon 1996; Benyon 1997). Neben dem Wachstum der Polizisten- und Beamtenclubs und ihrer Aufgaben zeigen diese Studien, dass die meisten Akteure in etlichen dieser Zusammenschlüsse gleichzeitig aktiv waren (Bigo 1996, S. 97-98). 
Die Literatur, die sich mit der Internationalisierung der inneren Sicherheit vor allem vor Beginn der 1990er-Jahre beschäftigt, steht allerdings vor zwei großen Schwierigkeiten. Zum einen bedingt die Informalität, dass es keine schriftlichen bindenden Regeln über Zahl, Formen und Inhalt der Zusammenarbeit gab, die Auskunft über ihre genaue Struktur geben könnte (Den Boer 2010, S. 52). Und die vorhandenen Schriftstücke - das ist die zweite Schwierigkeit - sind häufig geheim (Bigo 1996, S. 81). Dies erklärt auch, warum es zu dieser Phase der Polizeikooperation nur eine Handvoll politikwissenschaftlicher Studien gibt: Letztlich betätigten sich diese Autoren selbst als Ermittler, die versuchten aus den spärlichen, öffentlich zugänglichen Informationen, Memoiren von Polizisten und Interviews mit pensionierten Beamten ein Bild der damaligen Zusammenarbeit zu zeichnen. Lediglich eine kleine Anzahl von Polizeiforschern im weiteren Sinne äußerte sich auch zur EU, oft im Rahmen breiter angelegter Veröffentlichungen (Busch 1999, 1995; Aden 1998; Bigo 1996; Freestone 1981). Deshalb sind manche Studien eher der Bedrohungs- und Verschwörungsliteratur zuzuordnen (ein klassisches Beispiel ist Sterling 1981). Eine ganze Reihe wichtiger Dokumente aus dieser Zeit wurde mittlerweile von der britischen NGO „Statewatch“ auf ihrer Webseite veröffentlicht (www.statewatch.org). Nach Ablauf der 30-jährigen Sperrfrist öffnen sich langsam auch die Archive, so dass die empirische Forschung eine zunehmend breitere Grundlage hat.

Erklärt wird die Internationalisierung der inneren Sicherheit mit den Strategien und Problemwahrnehmungen bestimmter Akteure. Hier sind die bereits erwähnten Arbeiten von Didier Bigo, Malcolm Anderson oder Heiner Busch zu nennen, die explizit wie implizit - wesentlich zur Entwicklung der Informalitätsthese beigetragen haben und diese auch auf die Zusammenarbeit in der EU ausweiteten. In eine ähnliche Richtung argumentieren die Arbeiten des Soziologen Mathieu Deflem. Mittels eines auf Max Weber zurückgehenden Bürokratieansatzes analysiert er, wie nationale Polizeiapparate zunehmend autonomer wurden und so international kooperieren konnten (Deflem 2000, 2002). Ebenso gehören die Arbeiten der Gruppe um John Benyon (Benyon et al. 1993, 1994; Benyon 1997) in diese Strömung wie auch die bereits genannten Studien von Nadelmann, die das ausgeprägte Interesse der USA an internationaler Polizeikooperation herausstellen. Sie zeigen, dass der Einfluss der USA vor allem ab 1945 nicht nur in Europa, sondern weltweit ein Motor für die zunehmende Polizeikooperation war. Diese internationale Absicherung gelang, da die US-Präferenzen sehr ausgeprägt und ihre Durchsetzungsmacht hoch war (Cusack 1974), während viele Industriestaaten dem Problem bis in die 1960er-Jahre nur geringe Priorität zuwiesen, die Produzentenstaaten dagegen schwach und von den USA abhängig waren (vgl. im Überblick McAllister 2000). Diese Betonung der USA als der treibenden Kraft hinter der Internationalisierung der inneren Sicherheit allgemein ist weitgehender Konsens in der Literatur.

Die gleichen Muster werden auch in jüngster Zeit beobachtet, allerdings wird stärker explizit Bezug auf Netzwerkkonzepte genommen (vgl. z. B. Den Boer 2006, 2010; Den Boer et al. 2008 für den Bereich der EU). Dies jedoch ohne die - für die Analyse der Internationalisierung der inneren Sicherheit vielverspechenden - Möglichkeiten der IB-Forschung zu transgouvernementalen Netzwerken, die in jüngerer Zeit wieder belebt wurden, explizit aufzunehmen (Keohane u. Nye 
1974, S. 43; Slaughter 2004; Eilstrup-Sangiovanni 2005; 2009, S. 197-203). So basieren transgouvernementale Netzwerke nicht auf zwischenstaatlichen Verträgen, sondern auf informellen, nicht-bindenden Vereinbarungen. Die Akteure sind hochrangige Beamte der betroffenen Behörden, die Zusammenarbeit beruht ganz wesentlich auf Vertrauen, und gemeinsame Entscheidungen werden nur im Einvernehmen aller Beteiligter getroffen. Empirisch betont allerdings Monica den Boer (Den Boer 2010, S. 49) den politischen Spielraum, den diese Akteure haben, und zwar sowohl auf der horizontalen Ebene zwischen Staaten, als auch vertikal zwischen den Staaten und internationalen Institutionen. Wiederum treffen hier die Praktiker, hochstehenden Polizisten und Beamten der jeweiligen Ministerien auf bilateraler Ebene, in intergouvernementalen Gruppen oder Clubs zusammen, um sich über Möglichkeiten der Zusammenarbeit in den unterschiedlichsten Kriminalitätsbereichen auszutauschen - fern von Ratifikationszwängen, Sanktionsängsten und Implementierungsdruck.

Diese Polizistenclubs bilden aber nicht nur Entscheidungsnetzwerke, sondern auch „epistemic communities“ (Haas 1992), die die gemeinsame Problemwahrnehmung propagieren, wonach die transnationale Kriminalität der Zukunft nur durch grenzüberschreitende Zusammenarbeit bekämpft werden könne (Raustiala 2002). Durch diese können Polizisten und Polizeiexperten aufgrund ihrer fachlichen Autorität und ihres Wissens im Feld der Kriminalitätsbekämpfung umfassenden Einfluss auf politische Entscheidungen nehmen, die auf Dauer eine Konvergenz der Problemwahrnehmungen staatlicher Entscheidungsträger bewirken (Bigo 1996, 1999, 2000). Ein gutes Beispiel ist hier die späte Anerkennung in Frankreich, dass man ein nationales Drogenproblem habe. Denn trotz der French Connection gestand Frankreich dieses Problem erst Ende der 1960er-Jahre ein (Bergeron 1999; Bergeron u. Kopp 2002) - nachdem das Land bereits eine globale Drogenprohibitionskonvention (1961) unterzeichnet hatte und lange klar war, dass nicht nur der Drogentransit durch das eigene Land in die USA problematisch ist (Cusack 1974). Die vom französischen Präsidenten Georges Pompidou 1971 initiierte intergouvernementale „Pompidou-Gruppe“ (eigentlich Co-operation Group to Combat Drug Abuse and Illicit Trafficking in Drugs) - ein weiterer Polizistenclub - widmete sich in der Folge diesem Problem.

Diese implizite Thematisierung der Rolle von epistemic communities und die explizite Betonung von Problemdefinitionen ruft aus unserer Sicht förmlich nach konstruktivistischen Analysen. Betonen diese doch, wie Ideen von Kooperation in Expertennetzwerken entstehen und wie sich daraus eine normative Struktur bildet, die Zusammenarbeit und Internationalisierung selbst im Sicherheitsbereich fördert (vgl. Müller 2002, S. 380-382).

\section{Die Institutionalisierungsthese: Immer stärkere Institutionen in immer mehr Bereichen?}

Die Zahl und Wichtigkeit transgouvernementaler Netzwerke, in denen Polizeipraktiker und hohe Beamte flexibel und informell kooperieren, ohne dadurch in ihrer Handlungsfreiheit eingeschränkt oder Kontrollen durch Parlamente oder 
Gerichte unterworfen zu sein, ist allerdings nur eine Seite der Medaille. Parallel dazu wurde die internationale Kooperation in der inneren Sicherheit verrechtlicht, und es wurden formale internationale Organisationen gegründet. Die Literatur über diesen Entwicklungstrend überschneidet sich jedoch zumindest in den Anfangsjahren kaum mit der zur Informalitätsthese.

So passt die relativ gut erforschte Interpol zunächst noch zur Informalitätsthese. Die 1923 gegründete Commission Internationale de Police Criminelle, Vorläufer von Interpol, glich zumindest vor dem 2. Weltkrieg einem lockeren, informellen Zusammenschluss von Polizisten und Juristen (Anderson 1989, S. 39-41; Deflem 2002; Jensen 1981). Nach 1945 erfolgte der Wiederaufbau als International Criminal Police Organization, und seither fungiert diese unter dem Kürzel Interpol als globaler Rahmen für den Austausch von Informationen zwischen Polizeien (Anderson 1989; Anderson et al. 1995, S. 49). Dies erinnert sehr an die im vorigen Abschnitt beschriebenen Polizistenclubs. Allerdings hat sich Interpol durch eine Reihe von zwischenstaatlichen Verträgen immer mehr einer klassischen internationalen Organisation mit mittlerweile nahezu universeller Mitgliedschaft angenähert.

Erheblich stärker institutionalisiert und formalisiert ist die Drogenprohibition auf internationaler Ebene. Zwar begann sie informell: 1909 fand auf Initiative der USA mit der Internationalen Opium Kommission die erste globale Konferenz zum Thema Drogenprohibition in Shanghai statt (Musto 1973; Elvins 2003, S. 28; Briesen 2005, S. 13), die vorsah, eine gemeinsame rechtliche Basis für die Homogenisierung der nationalen Drogenpolitiken auf globaler Ebene zu schaffen. Trotz einer Vielzahl von internationalen Abkommen nahm die globale Drogenprohibition erst nach dem 2. Weltkrieg und der Gründung der Vereinten Nationen richtig Fahrt auf (Busch 1999; McAllister 2000; Bewley-Taylor 2003). Mit drei umfangreichen Abkommen, dem VN-Einheitsabkommen über die Betäubungsmittel (1961), der VN-Konvention über psychotrope Substanzen (1971) und dem VN-Übereinkommen gegen den unerlaubten Verkehr mit Suchtstoffen und psychotropen Stoffen (1988), wurde sukzessive das globale Drogenprohibitionsregime geschaffen.

Dieses Regime war schon sehr früh hochgradig verrechtlicht im Sinne der Typologie von Abbott et al. (2000; vgl. auch Herschinger et al. 2011). Seine Regeln sind sehr präzise, da erlaubte und verbotene Substanzen differenziert und in Form langer Listen festgelegt werden, sie verfügen über einen hohen Verpflichtungsgrad, da es sich um internationale Verträge handelt, und es findet eine gewisse Delegation von Exekutiv- wie von Regelsetzungsbefugnissen an andere einschlägige VNGremien statt (Jojarth 2009, S. 268). Hinsichtlich seiner institutionellen Stärke verpflichtet vor allem das Abkommen über psychotropische Substanzen von 1988 Staaten zur gegenseitigen Rechtshilfe - auch und gerade bei Auslieferungsfragen, zum Informationsaustausch und zur Kooperation in der Strafverfolgung (Busch 1999, S. 58; vgl. auch Bewley-Taylor 1999).

In den letzten zehn bis 15 Jahren wurde das Regime kontinuierlich durch die Stärkung seiner organisatorischen Komponente, dem UN Office on Drugs and Crime (UNODC), ausgebaut. Im Falle der EU kommt verschärfend hinzu, dass das Regime inzwischen Teil des acquis communitaire ist und so auch mit jeder 
EU-Erweiterung eine geographische Expansion erfährt (Boekhout van Solinge 2004, S. 56). Seit längerem gibt es massive Kritik an der Anti-Drogenpolitik des Regimes, das nationale Drogenpolitiken zu sehr auf das amerikanische Modell verpflichte (neben Ethan Nadelmann etwa von Gerber u. Jensen 2001), die sich jedoch weitgehend in spezialisierten Zeitschriften wie dem International Journal of Drug Policy abspielt (Bewley-Taylor u. Jelsma 2012).

Auf europäischer Ebene verlief die Entwicklung ähnlich. Nach dem Vertrag von Maastricht wurde mit der European Drug Unit jene Einheit geschaffen, die später zu Europol wurde (Occhipinti 2003). Diese wiederum ging auf TREVI zurück, das für die Anti-Drogenpolitik als eine Art Embryo europäischer Polizeikooperation fungierte. Es lässt sich somit festhalten, dass es sich sowohl beim globalen Anti-Drogenregime, als auch bei der europäischen Anti-Drogenpolitik nicht um informelle Zusammenschlüsse handelt, die Polizisten und Beamten weite Handlungsspielräume lassen, sondern um ausdifferenzierte und verpflichtende internationale Institutionen, die die Handlungsfähigkeit auch mächtiger Staaten massiv einschränken. Selbst die EU ist hier kein autonomer Akteur, sondern setzt das UN-Drogenregime letztlich nur um.

In diesem Zusammenhang ist das globale Anti-Geldwäscheregime ein interessanter Fall. Es beruht zwar letztlich auf dem hochgradig verrechtlichten VN-Drogenregime, dessen 1988er Konvention zentrale Abschnitte zur Bekämpfung der Geldwäsche enthält. Jedoch gingen wesentliche Entwicklungen von der OECD aus, deren Financial Action Task Force (FATF) den Kern des globalen Anti-Geldwäscheregimes bildet (Sharman 2011). Sein regulatorischer Kern beruht auf einer Reihe von Empfehlungen, die seit 1990 von der FATF erarbeitet wurden - also nicht auf völkerrechtlichen Verträgen, die von nationalen Parlamenten ratifiziert werden, sondern auf soft law. Aus dieser Perspektive steht das globale Geldwäscheregime im Einklang mit der Informalitätsthese und sollte den beteiligten Staaten große Autonomiespielräume bewahren. Wie die Literatur einhellig feststellt, ist dies jedoch kaum der Fall. In Europa beruht das Geldwäscheregime nicht alleine auf den Empfehlungen der OECD, sondern auch auf multilateralen Verträgen wie der Europaratskonvention von 1990 und der Geldwäscherichtlinie der EU, deren erste Fassung von 1991 bis heute zahlreiche Modifikationen erfahren hat (zuletzt 2010). Weiterhin geht die Reichweite des Regimes durch die Erweiterung der FATF-Mitgliedschaft und durch die Schaffung von „FATF-style regional bodies" in anderen Weltregionen mittlerweile deutlich über die OECD hinaus. Selbst wenn das Regime nicht wie in Europa stärker verrechtlicht ist, sondern nur auf Empfehlungen beruht, entfaltet es durch die Praxis der „multilateralen Überwachung“ erhebliche Wirkungen (Duyne u. Levi 2005; Gardner 2007).

Im Gegensatz dazu ist der Kampf gegen den Terrorismus der Fall einer nur teilweise gelungenen Internationalisierung. Nach den Anschlägen auf die Olympischen Spiele in München 1972 begannen die VN, an einer umfassenden Terrorismuskonvention zu arbeiten, u. a. um einen Rahmen für eine Reihe von früheren, teilweise noch auf den Völkerbund zurückgehenden und thematisch enger fokussierten Antiterror-Konventionen zu schaffen (Dubin 1991; Bassiouni 2001). Die Arbeiten wurden 1979 unterbrochenen und seit 1998 - erfolglos - weitergeführt. Ursache hierfür sind fundamentale Meinungsunterschiede über die Definition von 
„Terrorismus“ - ganz im Sinne des Klischees, dass des einen Terroristen des anderen Freiheitskämpfer ist. Das Thema der unterschiedlichen Definitionen von Terrorismus - ein klassisches konstruktivistisches Thema - wird in der Literatur intensiv diskutiert (vgl. z. B. Friedrichs 2006; Herschinger i. E.; Saul 2006). Auf VN-Ebene entstand seit 1972 zwar eine Reihe von Anti-Terrorkonventionen, die jedoch alle nur einen Teilaspekt des Problems abdecken und viele Exit-Optionen bieten. ${ }^{2}$

Aufgrund des Scheiterns der UN-Bemühungen versuchten die europäischen Staaten im Rahmen des Europarats, 1977 eine Anti-Terrorismuskonvention zu verabschieden. Aber auch diese brauchte nicht nur lange bis zum Inkrafttreten, sondern dokumentiert mit ihren vielen Ausnahmeklauseln und Austrittsmöglichkeiten, dass auch die westlichen Staaten mitnichten eine einheitliche Einschätzung des Phänomens „Terrorismus“ teilten (Lacoste 1982). Offenbar war auch der Europarat noch zu heterogen, um angesichts der unterschiedlichen Problemdefinitionen zu einem bindenden und effektiven Übereinkommen zu gelangen. Die Kooperation verlagerte sich deshalb in noch kleinere Foren: Die EU und den UN-Sicherheitsrat. In beiden Fällen verstärkten externe Stimuli (d. h. die Anschläge des 11. September 2001) die Kooperationsbereitschaft der Staaten deutlich. Die Bedeutung dieser „politics of the latest outrage“ (Wilkinson 2006, S. 166) wird in der Literatur durchgängig betont.

Innerhalb der VN hat sich inzwischen der exklusivere Sicherheitsrat zum Hauptakteur entwickelt. Er setzte nicht nur internationale Gerichtshöfe zur Verfolgung von Kriegsverbrechen ein (Schabas 2006), sondern wurde auch zu einem ebenso bedeutenden wie umstrittenen Akteur in der Antiterrorismuspolitik der VN, vor allem durch seine Resolutionen nach dem 11. September 2001 sowie die Aktivitäten des von ihm eingerichtete Counter-Terrorism Committee (Romaniuk 2010). Die Maßnahmen des Sicherheitsrates stützen sich auf Kapitel VII der VNCharta, die auch den Einsatz von militärischer Gewalt vorsieht. Hierzu existiert eine kaum noch überschaubare Literatur, die allerdings zum überwältigenden Teil von Juristen verfasst wird. Sie befasst sich vor allem mit der Bedeutung der Sicherheitsratsbeschlüsse für die Entwicklung des Völkerrechts, mit der Vereinbarkeit dieser Beschlüsse mit der Charta der VN, sowie mit ihrer Vereinbarkeit mit Grundrechten (vgl. im Überlick Krisch i. E.).

Die politikwissenschaftliche Literatur ist dagegen erheblich kleiner. Ein wesentlicher Diskussionsstrang ist hier die Beschäftigung mit der Autorität und der Legitimität des Sicherheitsrates, die allerdings meist sein gesamtes Aktivitätsspektrum umfasst und nicht nur den relativ kleinen Teil der Antiterrorismuspolitik (vgl. bspw. Hurd 2007; Cronin u. Hurd 2008). Hinsichtlich des Verhältnisses von informeller und institutionalisierter Kooperation liefert sie ein zwiespältiges Bild. Einerseits ist der Sicherheitsrat ein supranationaler Akteur, der Beschlüsse per Mehrheitsentscheidung (und unter dem Vorbehalt des Vetos der fünf ständigen Mitglieder) fasst, die für alle VN-Mitgliedstaaten gültig sind. Er kann so die Charta, die ein unvollständiger Vertrag ist, inhaltlich interpretieren und weiterentwickeln (Voeten 2008). In dieser Hinsicht ist der Sicherheitsrat stärker und auto-

2 Bislang wurden 14 Konventionen verabschiedet, vgl. dazu http://www.un.org/terrorism/ instruments.shtml (Aufruf 9.3.2012). 
nomer als alle anderen internationalen Akteure in der inneren Sicherheit: Er setzt Recht hierarchisch und delegiert Befugnisse an ein teilautonomes Gremium, das Counter-Terrorism Committee, das ganz in der Tradition informeller Kooperation weitgehend hinter verschlossenen Türen tagt. Andererseits sind die Beschlüsse des Sicherheitsrates häufig nicht sehr präzise und lassen viel Interpretationsspielraum. Obwohl der Sicherheitsrat auch den Einsatz von Gewalt anordnen kann, erzwingt er die Umsetzung seiner Beschlüsse meist nicht, sondern bedient sich weicher Steuerungsmittel wie Information oder des Aufbaus von Handlungskapazitäten, da die Ursache für Nichtbefolgung meist nicht im fehlenden Willen, sondern in den mangelnden Fähigkeiten der betroffenen Staaten liegt (Heupel 2007, 2008).

Der 11. September hatte ebenfalls Auswirkungen auf die Antiterrorismuskooperation in der EU. Hier wurden eine ganze Reihe von Maßnahmen verabschiedet, u.a. eine Definition von Terrorismus (2002/475/JHA). Besondere Aufmerksamkeit hat dabei der europäische Haftbefehl erfahren, der vor allem - aber nicht nur - zur Bekämpfung des Terrorismus eingeführt wurde. Im Kern ersetzt er die früheren Auslieferungsverfahren mit der Möglichkeit der politischen Entscheidung durch rein rechtliche Verfahren ohne Vetomöglichkeiten der politischen Entscheidungsträger. Für einige Beobachter war dies eine „Revolution“ (Plachta 2003). Der europäische Haftbefehl ist meilenweit von den früheren informellen Polizistennetzwerken entfernt, weil er von Staaten verlangt, auch eigene Staatsbürger im Grunde ohne politischen Ermessensspielraum an einen anderen Staat auszuliefern. Aufgrund seiner praktischen Relevanz gibt es über den Haftbefehl eine Flut von Literatur aus rechtswissenschaftlicher und Anwenderperspektive (z. B. Keijzer u. van Sliedregt 2009), allerdings nur wenig politikwissenschaftliche Analysen (Sievers 2011).

Nach dem Maastrichter Vertrag (1993), der mit der Schaffung eines „dritten Pfeilers" die Zusammenarbeit in Fragen der inneren Sicherheit eingeläutet hatte, verstärkte der Vertrag von Amsterdam (1999) diese, indem Teile des dritten Pfeilers in den (supranationalen) ersten Pfeiler übernommen wurden. Der Vertrag von Lissabon (2009) schließlich führte den größten Teil der Bestimmungen zur inneren Sicherheit in das „ordentliche Gesetzgebungsverfahren“ über, das ein Vorschlagsmonopol der Kommission, qualifizierte Mehrheit im Rat und Mitentscheidung des Europäischen Parlaments beinhaltet. Die Bestimmungen zur inneren Sicherheit sind nach Ansicht mancher Beobachter die am weitesten gehenden Reformen durch den Lissaboner Vertrag (Ladenburger 2008). Wie weit diese Institutionalisierung fortgeschritten ist, kann man zumindest indirekt daran ablesen, dass es mittlerweile einige Überblicksdarstellungen über die innere Sicherheit in der EU gibt, die allerdings meist von Juristen verfasst werden (Fletcher et al. 2008; Peers 2007; Mitsilegas 2009). Das Thema hat inzwischen auch einen festen Platz in den Annual Reviews des Journal of Common Market Studies (vgl. jüngst Monar 2011) und in den einschlägigen Lehrbüchern zur Politik in der EU (Hix u. Høyland 2011, Kap. 11; Lavenex 2010).

Auch wenn die Schaffung internationaler Institutionen im Bereich der inneren Sicherheit zunächst langsam und mit einigen Rückschlägen verlief, so ist die Entwicklung eine Bestätigung der Institutionalisierungsthese. Auch im Bereich der inneren Sicherheit werden internationale Regeln präziser, delegieren Staaten im- 
mer häufiger Ausführungs- und Rechtsetzungsbefugnisse an unabhängige Institutionen, steigt die Unabhängigkeit der Streitschlichtungsmechanismen von den Staaten und wird zumindest in der EU zunehmend mit Mehrheit entschieden. Kurz: internationale Institutionen werden stärker und zahlreicher und schränken staatlichen Handlungsautonomie ein (Herschinger et al. 2011).

Erklärungsbedürftig sind dabei sowohl die Ursachen der allgemeinen Entwicklung hin zu stärkeren Institutionen, als auch die Variation zwischen Politikbereichen und Weltregionen. Hierzu werden in der Literatur - die eher spärlich gesät ist - zwei Ansätze vertreten. Der erste ist die bereits erwähnte klassische funktionale Erklärung internationaler Kooperation, wonach Staaten durch institutionalisierte Zusammenarbeit negative Externalitäten einzuhegen suchen. Diesen Ansatz verfolgt der Großteil der einschlägigen Literatur, häufig mehr implizit als explizit (vgl. z. B. Knelangen 2001, 2006a, 2006b; Lange 1999). Man ist offenbar einhellig der Meinung, dass der Problemdruck durch transnationale Kriminalität in den letzten Jahrzehnten zugenommen hat, und dass deshalb mehr und stärkere internationale Institutionen geschaffen wurden. Auf der Makroebene erscheint dies auf den ersten Blick auch plausibel. Allerdings ist dies nur eine Makrokorrelation und keine Erklärung von Akteurspräferenzen, wie sie die liberale Außenhandelstheorie liefert (Moravcsik 2008). Über eine solche Makrokorrelation gehen nur wenige Autoren hinaus, so Jörg Friedrichs (2007), der die Präferenzen von vier westeuropäischen Staaten hinsichtlich der internationalen Bekämpfung von Terrorismus und Drogenhandel zu einem erheblichen Teil mit funktionalen Anreizen erklärt, allerdings auch normativen Faktoren eine gewisse Relevanz zuweist.

Ein weiterer, in der Literatur durchgängig genannter Grund für die Schaffung starker internationaler Institutionen ist der politische Druck der USA. Hier hat vor allem Nadelmann mit seiner Analyse zur Entstehung von globalen Prohibitionsregimen diese Erklärung untermauert (Nadelmann 1990; vgl. aber auch Bewley-Taylor 1999; Briesen 2005). Empirisch ist dies sicher zutreffend, theoretisch handelt es sich aber um einen Fall der oben diskutierten Problemdruckthese, wonach die USA besonders früh und besonders intensiv von negativen Externalitäten betroffen wurden bzw. dies so wahrgenommen haben (asymmetrische Betroffenheit). Damit einher geht die These, dass es den USA gelungen ist, ihre eigenen substantiellen und prozeduralen Präferenzen institutionell festzuschreiben und so veränderungsresistent zu machen. Oder in der Sprache der Principal-Agent-Theorie ausgedrückt: Durch Delegation an internationale Institutionen wurde ein „policy bias" im Sinne der USA geschaffen (Hawkins et al. 2006).

Während es zu den einzelnen Problembereichen wie Geldwäsche, organisierte Kriminalität, Terrorismus und Drogenprohibition eine ständig anwachsende Zahl von Untersuchungen gibt, so sind vergleichende Untersuchungen der Ursachen und Formen von Institutionalisierung im Bereich der inneren Sicherheit äußerst rar. Bestenfalls wird der Zusammenhang zweier Politikfelder (meist Terrorismus und Drogenhandel) analysiert (Björnehed 2004; Hollis 2007; Lee 2007). Lediglich die neuere Monographie von Christine Jojarth (2009) versucht, auf der Basis der „rational design“-Konzeption von Koremenos et al. (2001) den unterschiedlichen Institutionalisierungs- bzw. Verrechtlichungsgrad von verschiedenen Regelsystemen im Bereich der inneren Sicherheit (u. a. Drogenhandel und Geldwäsche) 
mit einem transaktionskostentheoretischen Ansatz zu erklären. Auch die Wahl unterschiedlicher Foren (UN, OECD, Europarat oder EU) wird in der Literatur wenig vergleichend untersucht. Nur die bereits erwähnte Monographie von Friedrichs (2007, S. 183) findet einen systematischen positiven Zusammenhang zwischen den Präferenzen westeuropäischer Staaten hinsichtlich der Tiefe, der geographischen Ausdehnung, sowie des substantiellen Umfangs der Zusammenarbeit. Diese Staaten wollen also möglichst globale starke Institutionen, anstatt eine substantiell umfangreiche und institutionell starke Zusammenarbeit in kleinem Kreise. Vergleichend sind auch die einschlägigen Arbeiten Peter Katzensteins. Er argumentiert, dass staatliche Präferenzen (untersucht werden Deutschland, Japan und teilweise die USA) hinsichtlich der Schaffung internationaler Institutionen im Bereich der inneren Sicherheit stark von kulturellen Normsystemen und Strukturen abhängen und somit scheinbar gleiche Probleme (wie etwa „Terrorismus“) durch diese Staaten auf völlig unterschiedliche Weise definiert und in politische Handlungsstrategien umgesetzt werden (Katzenstein 1996; 2003). Im Gegensatz zu den dominierenden funktionalistischen Ansätzen verfolgt Katzenstein somit einen explizit konstruktivistischen Ansatz.

Noch weiter in diese Richtung geht Anja Jacobi (2011), die auf den Weltgesellschafts-Ansatz der Stanford School um John Meyer zurückgreift (vgl. etwa Meyer et al. 1997). Jacobi zeichnet zum einen die Existenz und Verbreitung weltgesellschaftlicher normativer Standards anhand der Ratifikation globaler Abkommen in einer Reihe von Politikfeldern nach und erklärt zum anderen die Varianz zwischen diesen Feldern, indem sie zwischen substantiellen und prozeduralen Regeln und rationalisierenden und präskriptiven Regeln andererseits unterscheidet. Erstere beziehen sich dabei auf wissenschaftliche und professionelle Standards, die deshalb potentiell universell sind, während präskriptive Regeln stärker normativ und weniger „sachzwangorientiert“ sind und daher eher zur Fragmentierung führen. Laut Jacobi liegen im Fall des Terrorismus stärker präskriptive Regeln vor, dagegen sind die globale Verbreitung und Akzeptanz der internationalen Regeln im Bereich der Drogenbekämpfung und der Geldwäsche ein Fall rationalisierender Regeln. Diese Argumentation wird von der umfangreichen Literatur zum globalen Anti-Geldwäscheregime aufgenommen. So erklärt Jason Sharman, dass das Regime inhaltlich ein Fall von internationaler Policy-Diffusion ist, welches die Verpflichtung zum Kampf gegen Geldwäsche zu einer quasi-konsensualen globalen Norm macht (Sharman 2011). Seine Besonderheit besteht darin, dass sowohl die Kosten, als auch die tatsächliche Beobachtung von Geldströmen trotz dessen Protest dem Privatsektor übertragen und häufig durch strafrechtliche Bestimmungen sanktioniert werden (Favarel-Garrigues et al. 2011). Diese Tatsache mag auch zum Teil für die sehr erfolgreiche Umsetzung trotz eigentlich schwacher Verrechtlichung verantwortlich sein, da die Staaten so einerseits einer normativen Verpflichtung genügen können, die Kosten dafür jedoch nicht selbst tragen müssen. Aber auch für diese Entwicklung wird der Druck der USA als Erklärungsfaktor angeführt, denn, so betont Jacobi, dieser war gerade in der Anfangsphase des Geldwäscheregimes wesentlich für dessen Etablierung (Jacobi 2011, Kap. 7). 


\section{Die Parallelität von Informalität und Institutionalisierung und die Bedeutung des Konstruktivismus}

Bei der Durchsicht der Literatur zeigt sich, dass Informalität und Institutionalisierung keine konkurrierenden Erklärungsansätze sind, sondern unterschiedliche Ergebnisse der gleichen funktionalistischen Erklärung, wonach Staaten Externalitäten einzuhegen versuchen. Konkret stellt sich die Geschichte der Internationalisierung der inneren Sicherheit als ein langanhaltender Prozess dar, bei dem sich Informalität und Institutionalisierung teilweise zeitlich abwechseln, teilweise aber auch parallel verlaufen (Walker 2004). Nur einige wenige Studien betonen diese Parallelität: Malcolm Anderson und Kollegen (1995) versuchten mit der Unterscheidung zwischen „alten“ und „neuen“ Systemen der Polizeikooperation, das Zusammenspiel von Informalität versus Formalität einzufangen. Didier Bigo (1996) hat diese Unterscheidung ebenso aufgegriffen wie Monica den Boer (2010, S. 192).

Allerdings beziehen sich diese Studien ausschließlich auf die EU. Da sich der überwiegende Teil der Literatur einzelne Organisationen herausgreift, wird der Wechsel eines Themas zwischen verschiedenen Foren und der damit einhergehende Wandel zwischen Informalität und Institutionalisierung leicht übersehen. Typisch ist der Fall der Terrorismusbekämpfung, wo nach informellen Anfängen die im Rahmen der UN zunächst gescheiterte globale Institutionalisierung in verschiedenen anderen Foren (Sicherheitsrat, EU) mit unterschiedlichem Grad an Informalität und Institutionalisierung erneut versucht wurde. Entscheidend ist dabei, dass dieses Wechselspiel zwischen Informalität und Institutionalisierung nicht im Sinne eines „entweder/oder“ gemeint ist. Empirisch zeigt sich, dass informelle Kooperation nicht verschwindet, sobald Institutionen geschaffen wurden. Umgekehrt zeigt sich aber auch, dass informelle Kooperation eine wichtige Voraussetzung für die formelle Institutionalisierung war. Damit stellt aus unserer Sicht eine systematischere, vergleichende Untersuchung der Parallelität von informellen und institutionellen Formen der Internationalisierung der inneren Sicherheit ein Feld für zukünftige Forschung dar.

Interpol ist für dieses Zusammenspiel ein hervorragendes Beispiel, denn bis weit in die 1960er-Jahre war Interpol der formalen Organisationsform zum Trotz ein informeller Polizistenclub. Darin wird in der Literatur jedoch die entscheidende Leistung von Interpol damals gesehen: es war Treffpunkt und Mittel zur Herstellung wie Verstetigung von informellen Kontakten und vertrauensvollen Beziehungen zwischen hochrangigen Polizeibeamten (Gerspacher 2008, S. 174). Entscheidend für diesen hohen Stellenwert von Informalität ist, dass sich die Organisation zwar 1956 eine „Verfassung“ zu Regelung ihrer Arbeitsweise gegeben hat, aber bis heute nicht auf einem zwischenstaatlichen Vertrag beruht. Gerade mit Blick auf die Informationsübermittlung durch das Interpol-Kommunikationssystem bedeutete dies, dass Staaten nicht zum Austausch verpflichtet werden konnten bzw. dass sie durch ihre nationalen Büros selbst kontrollierten, welche Art und welches Ausmaß an Information sie übermitteln wollten (Anderson et al. 1995, S. 49-53).

Auch Europol kann als Beispiel für das Zusammenspiel von Informalität und Institutionalisierung zitiert werden. Seine Entwicklung war zunächst von sehr un- 
terschiedlichen Präferenzen der EU-Mitgliedstaaten geprägt, die die Entstehung einer funktionstüchtigen Organisation enorm erschwerten (Knelangen 2001, Kap. 8). Vor allem Deutschland stieß mit seiner Idee eines europäischen FBI mit Exekutivbefugnissen auf erbitterten Widerstand der Franzosen und Briten, die eine reine Vermittlungszentrale anstrebten. Erst nach dem Inkrafttreten des Vertrags von Amsterdam 1999 wurde Europol voll funktionstüchtig. Heute verfügt es über rund 700 Angestellte, ist jährlich in rund 12.000 Ermittlungen involviert, die grenzüberschreitende Straftaten (d. h. alle Formen von schwerer wie organisierter Kriminalität und Terrorismus) betreffen, und ist seit 2010 eine EU-Behörde, über deren Budget das Europäische Parlament wacht. Mit dieser starken Formalisierung der Institution sind ähnlich wie bei Interpol die informellen Praktiken nicht verschwunden. Nicht nur stellen die ca. 130 nationalen Verbindungsbeamten ein wichtiges Reservoir dar, auch die häufig von Praktikern geäußerte Unzufriedenheit mit den langwierigen Prozessen bei Europol (etwa im Rahmen der Gemeinsamen Ermittlungstruppen; Rijken 2006) führt dazu, dass informelle und bilaterale Netzwerke weiterhin eine zentrale Rolle spielen (Bigo 2000; Den Boer 2010).

Die Literatur thematisiert aber nicht explizit, unter welchen Bedingungen der jeweilige Typ der Zusammenarbeit - informell oder institutionalisiert - gewählt wird. Aus den vorliegenden Studien lässt sich aber schließen, dass Informalität vor allem dann gewählt wurde, wenn die Präferenzheterogenität der beteiligten Staaten groß war, keine großen Umsetzungsprobleme erwartet wurden und das gegenseitige Vertrauen gering war. Im Wesentlichen sind dies die Bedingungen, die die Literatur zu transgouvernementalen Netzwerken nennt (Keohane u. Nye 1974; Eilstrup-Sangiovanni 2005, 2009; Slaughter 2004). Die Geschichte der globalen Terrorismusbekämpfung ist hier ein besonders einschlägiges Beispiel. Internationalisierung war hingegen wahrscheinlicher, wenn eine allgemein akzeptierte Problemdefinition durch eine - eventuell hegemonial aufgezwungene - Problemperzeption vereinbart werden konnte (durchaus auch aufgrund einer gewissen Präferenzhomogenität der beteiligten Staaten), eine Implementation durch Staaten entscheidend für die Wirkungskraft einer Vereinbarung war oder auf bereits existierende Institutionen aufgebaut werden konnte. Hier lässt sich beispielhaft das Drogenprohibitionsregime nennen. Allerdings wird das Konzept der transgouvernementalen Netzwerke nur von wenigen Studien explizit eingesetzt (Newman 2008, 2011). Hier böte sich eine hervorragende Anknüpfungsmöglichkeit an die neuere Literatur über transgouvernementale Netzwerke, die explizit untersucht, wann Staaten netzwerkartigen Strukturen den Vorzug vor stärker institutionalisierten Formen der Kooperation geben (Kahler 2009).

Dieser Erklärungsstrang bewegt sich im Rahmen klassischer funktionalistischer, interdependenzorientierter Ansätze in den IB. Die inderdependenzorientierte Erklärung von Kooperation trifft allerdings auf besondere Schwierigkeiten aufgrund der besonderen Charakteristika des Politikfeldes innere Sicherheit. „Verbrechen“ sind wie kaum eine andere Kategorie gesellschaftliche Konstruktionen. Hinzu kommt die Dunkelziffer. Im Ergebnis ist das „reale“ Ausmaß des Problems und der bestehenden Inderdependenz nur sehr schwer feststellbar (stellvertretend für viele Maguire 2007, Kap. 1; Findlay 2000). Akteure reagieren vielmehr auf wahrgenommene und politisch postulierte Problemlagen. So wird die 
zunächst plausibel erscheinende These, dass der Abbau der Personenkontrollen innerhalb der EU und der Fall der Berliner Mauer zu einem Anstieg der grenzüberschreitenden Kriminalität geführt hätten und damit ursächlich für verstärkte EU-Zusammenarbeit waren, von Experten zurückgewiesen (Andreas u. Nadelmann 2006, S. 96; Estievenart 1995, S. 57).

Insofern verfügt eine konstruktivistische Erklärungsstrategie über erhebliche Stärken. In der auf innerstaatliche Politik bezogenen Kriminologie sind aus diesem Grund konstruktivistische Argumente weit verbreitet, seltsamerweise aber kaum in der Literatur zur internationalen Zusammenarbeit. Konstruktivismus wirkt jedoch geradezu prädestiniert für einen Bereich, in dem die gesellschaftliche Konstruktion bestimmter „Tatsachen“ offensichtlich zu sein scheint. Denn schon eine oberflächliche Betrachtung zeigt auf, dass bestimmte Taten manchmal als Verbrechen, manchmal als Bagatelle gewertet werden, dass sie in manchen Staaten verboten, in anderen dagegen erlaubt sind. Das hat Auswirkungen bis in die Kriminalstatistik. Es ist ein wesentlicher Grund für die fragmentierte Natur des VN-Antiterrorregimes (was ist Terrorismus?), ein wesentlicher Konfliktpunkt beim VN-Antidrogenregime (wie geht man mit Drogenabhängigen um?) (Herschinger 2011), zentrale Frage bei dem Umgang mit der transnationalen organisierten Kriminalität (was ist organisierte Kriminalität?) und wird auch bei der Diskussion um die Geldwäsche immer wieder thematisiert (Levi u. Reuter 2009; Hülsse 2007).

Diese Bedeutung der Wirklichkeitskonstruktionen zeigt sich vor allem mit Blick auf die Struktur der Kooperation - ein Potential, das bislang kaum genutzt wird. Die Literatur thematisiert zwar faktisch die Rolle von epistemic communities oder von Normunternehmern (Finnemore u. Sikkink 1998). Die Besonderheit im Bereich der inneren Sicherheit besteht darin, dass die Experten und Normunternehmer nicht in erster Linie unabhängige Wissenschaftler und zivilgesellschaftliche Akteure sind, sondern aus den Strafverfolgungsbehörden und Innenministerien kommen (Elvins 2003). Vor allem die bereits erwähnten Studien von Bigo sind hier einschlägig, der zeigt, wie unterschiedliche nationale Polizeiapparate aufgrund ihres Expertenwissens und ihrer Diskursmacht zu Motoren europäischer Kooperation im Sicherheitsbereich wurden (Bigo 1996) und sich durch ihre zunehmende grenzüberschreitende Kooperation (im territorialen wie administrativen Sinne) ein intensiv vernetztes Sicherheitsfeld etablieren konnte (Bigo 2000). Auch er verweist darauf, dass die zentralen Akteure Polizisten, Geheimdienstler, Beamte und Sicherheitsexperten aus Ministerien und internationale Organisationen sind. Ihre zunehmende Bedeutung manifestiert sich nicht nur auf europäischer Ebene, sondern zeigt sich auch in der Bekämpfung des internationalen Drogenhandels oder der Etablierung von sogenannten „Watch Lists“ von Terrorismusverdächtigen auf der Ebene der VN (Guild 2008). Neben Bigo betonen auch Nadelmann und Andreas (2006, S. 224-225) - wenn in geringerem Maße die Vorteile eines konstruktivistischen Ansatzes.

Der Konstruktivismus wird aber bei der Diskussion theoretischer Zugänge gar nicht erst erwähnt (vgl. Den Boer 2010, S. 50-51) oder taucht nur am Rande auf, so dass eine konsequente Rückbindung an diese IB-Theorie nicht stattfindet. Hier liegt ein zentrales Desiderat für die Analyse der Internationalisierung der inneren 
Sicherheit. Zudem könnten auch Ansätze aus der neueren Sicherheitsforschung noch stärker einbezogen werden, denn gerade die Securitization-Forschung (grundlegend Buzan et al. 1998) kann treffende Einblicke bieten. Es ist wieder Didier Bigo, der in einer älteren Studie (2000) erste Untersuchungsfährten in diese Richtung legte, wenn er erklärt, dass die zunehmend übereinstimmenden Problemdefinitionen - der organisierten Kriminalität, des Terrorismus - sich im weiteren Verlauf immer stärker homogenisierten und die Forderung nach außergewöhnlichen sicherheitspolitischen Maßnahmen wie bspw. die Verknüpfung von Datenbanken, Rasterfahndungen oder erweiterte Zeiten für Untersuchungshaft, die auf Migranten, Terroristen und Drogenkriminelle gleichermaßen zur Anwendung kommen sollten, enorm verschärften. Eine erste Untersuchung der Internationalisierung der inneren Sicherheit aus Perspektive der Securitization-Theorie liefert der Beitrag von Thierry Balzacq zur EU und Europol (Balzacq 2008), der auch die gesellschaftspolitischen Implikationen der Kooperation kritisch beleuchtet.

Unser Blick durch die Literatur zur internationalen Kooperation im Bereich der inneren Sicherheit zeigt somit, dass das Politikfeld kein Sonderfall ist, sondern mit den üblichen Mitteln zentraler IB-Theorien analysiert werden kann - auch wenn dies die vorliegende Literatur nur in Ansätzen tut. Gerade weil das Politikfeld eng an Staatlichkeit, Gewaltmonopol, Herrschaftssicherung und Grundfreiheiten verknüpft ist und in den letzten 20 Jahren einen dramatischen Aufschwung erfahren hat, ist die Dominanz deskriptiver und policy-orientierter Arbeiten wie die geringe Anbindung an IB-Debatten bedauerlich. Dabei haben sowohl funktionalistische Ansätze, die die Gründe für die Wahl bestimmter Kooperationsformen (Netzwerke oder Institutionen), als auch konstruktivistische Ansätze, die der hohen Bedeutung von Wirklichkeitskonstruktionen gerade in diesem Politikfeld Rechnung tragen, ein erhebliches Potential. Dieses Potential sollte für ein so wichtiges Politikfeld genutzt werden, das ebenso viel Aufmerksamkeit verdient hat wie andere, teilweise überforschte Politikfelder.

\section{Literatur}

Abbott, Kenneth O. W., Robert Keohane, Andrew Moravcsik, Anne-Marie Slaughter und Duncan Snidal. 2000. The Concept of Legalization. International Organization 54:401-419.

Aden, Hartmut. 1998. Polizeipolitik in Europa. Eine interdisziplinäre Studie über die Polizeiarbeit in Europa am Beispiel Deutschlands, Frankreichs und der Niederlande. Opladen: Westdeutscher Verlag.

Anderson, Malcolm. 1989. Policing the World. Interpol and the Politics of Police Cooperation. London: Clarendon Press.

Anderson, Malcolm, Monica den Boer, Peter J. Cullen, William Gilmore, Charles Raab und Neil Walker. 1995. Policing the European Union. Oxford: Clarendon Press.

Andreas, Peter, und Ethan Nadelmann. 2006. Policing the Globe. Criminalization and Crime Control in International Relations. Oxford u. a.: Oxford University Press.

Balzacq, Thierry. 2008. The Policy Tools of Securitization: Information Exchange, EU Foreign and Interior Policies. Journal of Common Market Studies 46:75-100. 
Bassiouni, M. Cherif. 2001. International Terrorism: Multilateral Conventions (19372001). New York: Transnational Publishers.

Bayley, David H. 1985. Patterns of Policing. A Comparative International Analysis. New Brunswick: Rutgers University Press.

Benyon, James. 1997. The Developing System of Police Cooperation in the European Union. In Crime and Law Enforcement in the Global Village, Hrsg. William F. McDonald, 103-121. Nunn Hall/Cincinnati: ACJS.

Benyon, James, Lynne Turnbull, Andrew Willis und Rachel Woodward. 1994. Understanding Police Cooperation in Europe: Setting a Framework for Analysis. In Policing Across National Boundaries, Hrsg. Malcolm Anderson und Monica Den Boer, 46-65. London/ New York: St. Martins Press.

Benyon, James, Lynne Turnbull, Andrew Willis, Rachel Woodward und Adrian Beck. 1993. Police Co-operation in Europe. An investigation. University of Leicester: Centre for the Study of Public Order.

Benyon, John. 1996. The Politics of Police Co-operation in the European Union. International Journal of the Sociology of Law 24: 353-379.

Bergeron, Henri. 1999. L'Etat et la Toxicomanie. Histoire d'une singularité française. Paris: Presses Universitaires de France.

Bergeron, Henri, und Pierre Kopp. 2002. Policy Paradigms, Ideas, and Interests: The Case of the French Public Health Policy towards Drug Abuse. The Annals of The American Academy of Political and Social Science 582:37-48.

Bewley-Taylor, David, und Martin Jelsma. 2012. Regime Change: Re-visiting the 1961 Single Convention on Narcotic Drugs. The International Journal on drug policy 23:72-81.

Bewley-Taylor, David R. 1999. The United States and international drug control, 19091997. London/New York: Pinter.

Bewley-Taylor, David R. 2003. Challenging the UN Drug Control Conventions: Problems and Possibilities. The International Journal of Drug Policy 14:171-179.

Bigo, Didier. 1996. Polices en réseaux: l'expérience européenne. Paris: Presses de la Fondation Nationale des Sciences Politiques.

Bigo, Didier. 1999. Vers Une Europe Des Polices? Revue française d'administration politique 91:471-482.

Bigo, Didier. 2000. Liason officers in Europe. New officers in the European security field. In Issues in Transnational Policing, Hrsg. John W. E. Sheptycki, 67-99. London/New York: Routledge.

Björnehed, Emma. 2004. Narco-Terrorism: The Merger of the War on Drugs and the War on Terror. Global Crime 6:305-324.

Boekhout van Solinge, Tim. 2004. Dealing with Drugs in Europe. An Investigation of European Drug Control Experiences: France, the Netherlands and Sweden. The Hague: BJu Legal Publishers.

Boldt, Hans. 2007. Geschichte der Polizei in Deutschland. In Handbuch des Polizeirechts: Gefahrenabwehr, Strafverfolgung, Rechtsschutz, Hrsg. Hans Lisken und Erhard Denninger, 1-41. München: Beck.

Briesen, Detlef. 2005. Drogenkonsum und Drogenpolitik in Deutschland und den USA. Ein historischer Vergleich. Frankfurt/New York: Campus Verlag.

Busch, Heiner. 1995. Grenzenlose Polizei? Neue Grenzen und polizeiliche Zusammenarbeit in Europa. Münster: Westfälisches Dampfboot. 
Busch, Heiner. 1999. Polizeiliche Drogenbekämpfung. Eine internationale Verstrickung Münster: Westfälisches Dampfboot.

Buzan, Barry, Ole Wrever und Jaap de Wilde. 1998. Security. A New Framework for Analysis. Boulder: Lynne Rienner.

Cronin, Bruce, und Ian Hurd (Hrsg.). 2008. The UN Security Council and the Politics of International Authority. Abingdon/New York: Routledge.

Cusack, John T. 1974. Response of the Government of France to the International Heroin Problem. In Drugs, Politics, and Diplomacy: The International Connection, Hrsg. Luiz R. S. Simmons und Abdul A. Said, 229-256. London: Sage.

Deflem, Mathieu. 2000. Bureaucratization and Social Control: Historical Foundation of International Police Cooperation. Law and Society Review 34:739-778.

Deflem, Mathieu. 2002. Policing World Society: Historical Foundations of International Police Cooperation. Oxford: Oxford University Press.

Den Boer, Monica. 2005. Copweb Europe: Venues, Virtues and Vexations of Transantional Policing. In Transnational European Union: Towards a Common Political Space, Hrsg. Wolfram Kaiser und Peter Starie, 191-209. London: Routledge.

Den Boer, Monica. 2006. Fusing the Fragments: Challenges for EU Internal Security Governance on Terrorism. In International Terrorism. A European Response to a Global Threat?, Hrsg. Dieter Mahncke und Jörg Monar, 83-111. Brüssel: Peter Lang.

Den Boer, Monica. 2010. Towards a governance model of police cooperation in Europe: the twist between networks and bureaucracies In International Police Cooperation. Emerging issues, theory and practice, Hrsg. Frédéric Lemineux, 42-61. Culompton: Willan Publishing.

Den Boer, Monica, Claudia Hillebrand und Andreas Nölke. 2008. Legitimacy under Pressure: The European Web of Counter-Terrorism Networks. Journal of Common Market Studies 46:101-124.

Dubin, Martin David. 1991. International Terrorism: Two League of Nations Conventions, 1934-1937. Millwood: Kraus International Publiations.

Duyne, Petrus C. van, und Michael Levi. 2005. Drugs and Money. Managing the Drug Trade and Crime-Money in Europe. London/York: Routledge.

Eilstrup-Sangiovanni, Mette. 2005. Transnational Networks and New Security Threats. Cambridge Review of International Affairs 18:7-13.

Eilstrup-Sangiovanni, Mette. 2009. Varieties of Cooperation. Government Networks in International Security. In Networked politics: agency, power, and governance, Hrsg. Miles Kahler, 194-227. Ithaca: Cornell University Press.

Elvins, Martin. 2003. Anti-Drug Policies of the European Union. Transnational DecisionMaking and the Politics of Expertise. Basingstoke: Palgrave Macmillan.

Emsley, Clive. 1996. The English Police: A Political and Social History. London: Longman. Estievenart, Georges. 1995. The European Community and the Global Drug Phenomenon. Current Situation and Outlook. In Policies and Strategies to Combat Drugs in Europe, Hrsg. Georges Estievenart, 50-95. Florence: Martinus Nijhoff Publishers.

Favarel-Garrigues, Gilles, Thierry Godefroy und Pierre 2011 Lascoumes. 2011. Reluctant Partners? Banks in the Fight Against Money Laundering and Terrorism Financing in France. Security Dialogue 42:179-196.

Findlay, Mark 2000. 2000. The Globalisation of Crime. Understanding Transitional Relationships in Context. Cambridge: Cambridge University Press. 
Finnemore, Martha, und Kathryn Sikkink. 1998. International Norm Dynamics and Political Change. International Organization 52:887-918.

Fletcher, Maria, Robin Lööf und Bill Gilmore. 2008. EU Criminal Law and Justice. Cheltenham: Edward Elgar.

Freestone, David. 1981. Legal Responses to Terrorism: Towards European Cooperation. In Terrorism: A Challenge to the State, Hrsg. Juliet Lodge, 195-224. Oxford: Martin Robertson.

Friedrichs, Jörg. 2006. Defining the International Public Enemy: The Political Struggle behind the Legal Debate on International Terrorism. Leiden Journal of International Law 19:69-91.

Friedrichs, Jörg. 2007. Fighting Terrorism and Drugs. Europe and International Police Cooperation. London: Routledge.

Friman, Richard H. 1996. NarcoDiplomacy: Exporting the US War on Drugs. Ithaca: Cornell University Press.

Gardner, Kathryn L. 2007. Fighting Terrorism the FATF Way. Global Governance 13:325345.

Gerber, Jurg, und Eric L. Jensen (Hrsg.). 2001. Drug War, American Style: The Internationalization of Failed Policy and its Alternatives. New York: Garland.

Gerspacher, Nadia. 2008. The History of International Police Cooperation: a 150-year Evolution in Trends and Approaches. Global Crime 9:169-184.

Guild, Elspeth. 2008. The Uses and Abuses of Counter-Terrorism Policies in Europe: The Case of the 'Terrorist Lists'. Journal of Common Market Studies 46:173-193.

Haas, Peter (Hrsg.). 1992. Knowledge, power, and international policy coordination. Cambridge: Cambridge University Press.

Hawkins, Darren G., David A. Lake, Daniel L. Nielson und Michael J. Tierney. 2006. Delegation and Agency in International Organizations. Cambridge/New York: Cambridge University Press.

Herschinger, Eva. 2011. Constructing Global Enemies. Hegemony and Identity in International Discourses on Terrorism and Drug Probibition. Abingdon/New York: Routledge.

Herschinger, Eva. Im Erscheinen. A Battlefield of Meanings. The Struggle for Identity in the UN-Debates on a Definition of International Terrorism. Terrorism and Political Violence.

Herschinger, Eva, Markus Jachtenfuchs und Christiane Kraft-Kasack. 2011. Scratching the Heart of the Artichoke? How International Institutions and the European Union Constrain the State Monopoly of Force. European Political Science Review 3:445-468.

Heupel, Monika. 2007. Adapting to Transnational Terrorism. The UN Security Council's Evolving Approach to Terrorism. Security Dialogue 38:477-499.

Heupel, Monika. 2008. Combining Hierarchical and Soft Modes of Governance. The UN Security Council's Approach to Terrorism and WMD Proliferation after 9/11. Cooperation and Conflict 34:7-29.

Hix, Simon, und Bjørn Høyland. 2011. The Political System of the European Union. Basingstoke: Palgrave Macmillan.

Hollis, André D. 2007. Narcoterrorism: A Definitional and Operational Transnational Challenge. In Transnational threats : smuggling and trafficking in arms, drugs, and human life, Hrsg. Kimberley L. Thachuk, 23-35. Westport: Praeger Security International.

Hülsse, Rainer. 2007. Creating Demand for Global Governance. The Making of a Global Moneylaundering Problem. Global Society 21:155-178. 
Hurd, Ian. 2007. After Anarchy: Legitimacy and Power in the United Nations Security Council. Princeton: Princeton University Press.

Jacobi, Anja P. 2011. Common Goods and Evils? The Formation of Global Crime Governance. Darmstadt: Habilitationsschrift, TU Darmstadt.

Jensen, Richard B. 1981. The International Anarchist Conference of 1989 and the Origins of Interpol. Journal of Contemporary History 16: 323-347.

Jojarth, Christine. 2009. Crime, War, and Global Trafficking. Cambridge: Cambridge University Press.

Kahler, Miles. 2009. Networked Politics: Agency, Power, and Governance. In Networked Politics: Agency, Power, and Governance, Hrsg. Miles Kahler, 1-20. Ithaca: Cornell University Press.

Katzenstein, Peter J. 1996. The Culture of National Security: Norms and Identity in World Politics. New York: Columbia University.

Katzenstein, Peter J. 2003. Same war - Different Views. Germany, Japan, and Counterterrorism. International Organization 57:731-760.

Keijzer, Nico, und Elies van Sliedregt (Hrsg.). 2009. The European Arrest Warrant in Practice. Den Haag: T.M.C. Asser Press.

Keohane, Robert O. 1984. After Hegemony: Cooperation and Discord in the World Political Economy. Princeton: Princeton University Press.

Keohane, Robert O., und Joseph S. Nye. 1974. Transgovernmental Relations and International Organizations. World Politics 27:39-62.

Knelangen, Wilhelm. 2001. Das Politikfeld innere Sicherheit im Integrationsprozess. Opladen: Leske + Budrich.

Knelangen, Wilhelm. 2006a. Die Europäische Union und die Bekämpfung des Terrorismus. In Europäisierung und Internationalisierung der Polizei, Hrsg. Martin H. W. Möllers und Robert Chr. van Ooyen, 69-80. Frankfurt a. M.: Verlag für Polizeiwissenschaft/Dr. Clemens Lorei.

Knelangen, Wilhelm. 2006b. Die innen- und justizpolitische Zusammenarbeit der EU und die Bekämpfung des Terrorismus. In Die Europäische Union im Kampf gegen den Terrorismus: Sicherheit vs. Freiheit, Hrsg. Erwin Müller und Patricia Schneider, 140-162. Baden-Baden: Nomos.

Knöbl, Wolfgang. 2008. Polizei und Herrschaft im Modernisierungsprozeß: Staatsbildung und innere Sicherheit in Preußen, England und Amerika 1700-1914. Frankfurt a. M.: Campus Verlag.

Koremenos, Barabara, Charles Lipson und Duncan Snidal. 2001. The Rational Design of International Institutions. International Organization 55:761-799.

Krisch, Nico. Im Erscheinen. Chapter VII Powers. The General Framework. In The Charter of the United Nations: A Commentary, Hrsg. Bruno Simma, et al. Oxford: Oxford University Press.

Lacoste, Ilse. 1982. Die Europäische Terrorismus-Konvention: eine Untersuchung des Europäischen Übereinkommens zur Bekämpfung des Terrorismus vom 27. Januar 1977 im Vergleich mit ähnlichen internationalen Abkommen und unter Berücksichtigung des schweizerischen Rechts. Zürich: Schulthess.

Ladenburger, Clemens. 2008. Police and Criminal Law in the Treaty of Lisbon. European Constitutional Law Review 4:20-40. 
Lange, Hans-Jürgen. 1999. Innere Sicherheit im politischen System der Bundesrepublik Deutschland. Opladen: Leske + Budrich.

Lavenex, Sandra. 2010. Justice and Home Affairs. Communitarization with Hesitation. In Policy-Making in the European Union, Hrsg. Helen Wallace et al., 457-477. Oxford: Oxford University Press.

Lee, Gregory D. 2007. The Global Drug Trade and its Nexus to Terrorism. In Countering Terrorism and Insurgency in the 21st century: International perspectives, Hrsg. James J. F. Forest, 178-195. Westport: Praeger Security International.

Levi, Michael, und Paul Reuter. 2009. Money Laundering. In The Oxford Handbook of Crime and Public Policy, Hrsg. Michael Tonry, 356-380. Oxford: Oxford University Press.

Maguire, Mike. 2007. Crime Data and Statistics. In The Oxford Handbook of Criminology, Hrsg. Mike Maguire, et al., 241-301. Oxford: Oxford University Press.

McAllister, William B. 2000. Drug Diplomacy in the Twentieth Century. An International History. London/New York: Routledge.

Meyer, John W., John Boli und George M. Thomas. 1997. World Society and the NationState. American Journal of Sociology 103:144-181.

Mitsilegas, Valsamis. 2009. EU Criminal Law. Oxford: Hart.

Monar, Jörg. 2011. Justice and Home Affairs. Journal of Common Market Studies Annual Review 49:145-164.

Moravcsik, Andrewb. 2008. The New Liberalism. In The Oxford Handbook of International Relations, Hrsg. Christian Reus-Smit und Duncan Snidal, 234-254. Oxford u. a.: Oxford University Press.

Müller, Harald. 2002. Security Cooperation. In Handbook of International Relations, Hrsg. Walter Carlsnaes et al., 369-391. London u. a.: Sage.

Musto, David. 1973. The American Disease. Origins of Narcotics Control. New Haven, London: Yale University Press.

Nadelmann, Ethan A. 1990. Global prohibition regimes: the evolution of norms in international society. International Organization 44:479-526.

Nadelmann, Ethan A. 1993a. The Internationalization of Police Cooperation in Western Europe. University Park: Pensylvannia University Press.

Nadelmann, Ethan A. 1993b. U.S. Police Activities in Europe. In The Internationalization of Police Cooperation in Western Europe, Hrsg. Fijnaut, Cyrille, 135-154. Deventer/ Boston: Kluwer Law and Taxation Publishers.

Nadelmann, Ethan A. 1995. The DEA in Europe. In Undercover: Police Surveillance in Comparative Perspective, Hrsg. Cyrille Fijnaut und Gary Marx, 269-289. The Hague: Kluwer.

Newman, Abraham L. 2008. Building Transnational Civil Liberties. Transgovernmental Entrepreneurs and the European Data Privacy Directive. International Organization 62:103-130.

Newman, Abraham L. 2011. Transatlantic Flight Fights. Multi-level Governance, Actor Entrepreneurship and International Anti-terrorism Cooperation. Review of International Political Economy 18:481-505.

Occhipinti, John D. 2003. The Politics of EU Police Cooperation. Toward a European FBI? Boulder: Lynne Rienner.

Peers, Steve. 2007. EU Justice and Home Affairs Law. Oxford: Oxford University Press. 
Plachta, Michael. 2003. European Arrest Warrant. Revolution in Extradition? European Journal of Crime, Criminal Law and Criminal Justice 11:178-94.

Raustiala, Kal. 2002. The Architecture of International Cooperation: Transgovernmental Networks and the Future of International Law. Virginia Journal of International Law 43:1-92.

Rijken, Conny. 2006. Joint Investigation Teams: principles, practice, and problems. Lessons learnt from the first efforts to establish a JIT. Utrecht Law Review 2:99-118.

Romaniuk, Peter. 2010. Multilateral Counter-Terrorism: The global politics of cooperation and contestation. New York: Routledge.

Saul, Ben. 2006. Defining Terrorism in International Law. Oxford: Oxford University Press.

Schabas, William A. 2006. The UN International Criminal Tribunals. The Former Yugoslavia, Rwanda and Sierra Leone. Cambridge: Cambridge University Press.

Sharman, Jason C. 2011. Power in Diffusion. Making Anti-Money Laundering Global. Ithaca: Cornell University Press.

Sheptycki, James W. E. 2002. In Search of Transnational Policing. Towards a Sociology of Global Policing. Aldershot: Ashgate.

Sievers, Julia. 2011. Mutual Recognition in EU Justice and Home Affairs. The Case of the European Arrest Warrant. Bremen: Ph.D. thesis, University of Bremen.

Slaughter, Anne-Marie. 2004. A new world order. Princeton: Princeton University Press.

Sterling, Claire. 1981. The Terror Network. The Secret War of International Terrorism. New York: Henry Holt \& Co.

Voeten, Eric. 2008. Delegation and the Nature of Security Council Authority. In The UN Security Council and the Politics of International Authority: Law, Politics and Power, Hrsg. Bruce Cronin und Ian Hurd, 43-56. Abingdon/New York: Routledge

Walker, Neil. 2004. In Search of the Area of Freedom, Security and Justice: A Constitutional Odyssey. In Europe's Area of Freedom, Security, and Justice, Hrsg. Neil Walker, 3-40. Oxford: Oxford University Press.

Weber, Max. 1980. Wirtschaft und Gesellschaft. Grundriss der verstehenden Soziologie. 5. Aufl. Tübingen: J.C.B. Mohr (Paul Siebeck).

Wilkinson, Paul. 2006. Terrorism versus Democracy: the Liberal State Response. London/ New York: Routledge.

Wolf, Klaus Dieter. 2000. Die neue Staatsräson. Zwischenstaatliche Kooperation als Demokratieproblem in der Weltgesellschaft. Plädoyer für eine geordnete Entstaatlichung des Regierens jenseits des Staates. Baden-Baden: Nomos.

\section{Autorlnnenangaben:}

Prof. Dr. Markus Jachtenfuchs, Hertie School of Governance, Friedrichstraße 180, 10117 Berlin, jachtenfuchs@hertie-school.org

[Korrespondenzautor]

Dr. Eva Herschinger,

Universität der Bundeswehr München, Institut für Politikwissenschaft, Werner-Heisenberg-Weg 39, 85577 Neubiberg, eva.herschinger@unibw.de 